\title{
Evaluation of AH36 microalloyed steel welded joint by submerged arc welding process with one and two wires
}

\author{
Anderson Clayton Nascimento Ribeiro ${ }^{a}$, Hani Henein ${ }^{b}$, Douglas G. Ivey ${ }^{b}$, Sergio Duarte Brandi ${ }^{a}$ \\ ${ }^{a}$ Department of Metallurgical Engineering, University of São Paulo - USP, Av. Professor Mello Moraes, \\ 2463, CEP: 05508-030, São Paulo, SP, Brazil \\ ${ }^{b}$ Department of Chemical and Materials Engineering, University of Alberta, \\ Edmonton, Alberta, Canada
}

Received: September 7, 2015; Accepted: November 26, 2015

\begin{abstract}
Shipbuilding is going through a period of revitalization, growth and technological advancement. One component of these innovations is to improve welding techniques and materials for optimizing processing time, reducing costs and to improve properties and ship performance. The aim of the present work is to evaluate the welding of microalloyed shipbuilding steel, AH36, using the submerged arc welding (SAW) process with one and two wires. The mechanical properties of the welded joint will be presented using microhardness and Charpy V-notch testing. For metallographic characterization, the base metal and welded joint were etched with $2 \%$ Nital and Klemm's I reagent. Scanning electron microscopy, X-ray diffraction and colored etching were used to quantify and verify the presence of martensite and retained austenite, the MA microconstituent. The results obtained from Charpy impact tests and another mechanical test can be correlated with the associated microstructure.
\end{abstract}

Keywords: AH36 steel, shipbuilding steel, Tandem SAW, MA microconstituent

\section{Introduction}

The shipbuilding industry requires technological advances, such as research in welding techniques and appropriate materials to reduce processing time and costs without impairing the desired mechanical properties. Recently, researchers around the world have focused on research and development of high strength low alloy steels (HSLA), as well as the investigation of various factors influencing their weldability.

Steel plates produced by thermo-mechanical control processing (TMCP) combined with various microalloyed elements have provided an effective solution to those requirements, offering better combinations of strength and toughness without impairing the weldability ${ }^{1,2}$. Welding accounts for a one third of all services performed in the shipbuilding industry, with submerged arc welding (SAW) being one of the most widely used processes ${ }^{3}$. Tandem submerged arc welding has been studied to increase the welding productivity.

The tandem technique process uses two or more wires aligned in the welding direction to increase the deposition rate and welding speed ${ }^{4}$.The tandem SAW has been used as an alternative to the conventional single wire SAW process, mainly in plates of medium and large thickness (greater or equal to $20 \mathrm{~mm})^{5}$ Tandem SAW creates stronger and tougher weld joints due to a suitable thermal cycle in the heat affected zone, when compared with thermal cycles for a one wire welding process. The purpose of this work is to evaluate the welding of microalloyed shipbuilding steel, AH36, produced via TMCP, using flux cored arc welding

*e-mail: andersoncnribeiro@usp.br, anribeir@ualberta.ca
(FCAW) at the root and filling with SAW with one wire and with two wires.

\section{Experimental Procedure}

The base metal used in this work was microalloyed shipbuilding steel, AH36, produced via TMCP, with a $20 \mathrm{~mm}$ thickness. Table 1 shows the chemical composition and the mechanical properties of the base metal based on the ASTM A131 standard ${ }^{6}$ and the equivalent carbon in accordance with IIW. The geometry of the joints is shown in Figure 1.

Both single wire (S-SAW) and two wires tandem SAW (T-SAW) were used in this work. The root pass, for both welds used the flux-cored arc welding E71T-1 filler metal with a diameter of $1.20 \mathrm{~mm}^{7}$ and $100 \% \mathrm{CO}_{2}$ as the shielding gas. The chemical compositions and mechanical properties of the FCAW root pass consumable and the SAW electrode are presented in Table 2 and Table 3 . The welding parameters are typical of industrial practice and are shown in Table 4 and Table 5. The inter-pass temperature was controlled to be less than $250^{\circ} \mathrm{C}$. The heat input was calculated with the conventional heat input equation using a coefficient of heat transfer into the plate of $0.95^{8}$.

Optical microscopy characterization was done by polishing and etching the samples using $2 \%$ Nital and Klemm 1. This reagent colors the microstructure and reveals martensite and austenite microconstituents (MA) as white and ferrite as blue. An image analysis program was used to measure the MA volume fraction. A scanning electron microscope (SEM) was used to examine the fracture surface of various specimens. 
Table 1: Chemical composition and mechanical properties of the base metal.

\begin{tabular}{|c|c|c|c|c|c|c|c|}
\hline \multicolumn{8}{|c|}{ Chemical composition (wt\%) } \\
\hline Element & $\mathbf{C}$ & Mn & Si & $\mathbf{P}$ & $\mathrm{Cr}$ & Al & Nb \\
\hline Base metal & 0.153 & 1.092 & 0.216 & 0.012 & 0.02 & 0.03 & 0.01 \\
\hline ASTM A131 & 0.18 & $0.90-1.60$ & $0.1-0.50$ & 0.035 & 0.2 & 0.015 & $0.02-0.05$ \\
\hline \multicolumn{8}{|c|}{ Mechanical properties from ASTM A131 } \\
\hline \multicolumn{4}{|c|}{ Yield stress (MPa) } & \multicolumn{4}{|c|}{$355 \mathrm{~min}}$. \\
\hline & \multicolumn{3}{|c|}{ Ultimate strength $(\mathrm{MPa})$} & \multicolumn{4}{|c|}{$490-620$} \\
\hline & \multicolumn{3}{|c|}{ Elongation (\%) } & \multicolumn{4}{|c|}{15} \\
\hline & \multicolumn{3}{|c|}{ Absorbed energy $(\mathrm{J}) 0^{\circ} \mathrm{C}$} & \multicolumn{4}{|c|}{34} \\
\hline
\end{tabular}

Carbon Equivalent $=0.38 \%$

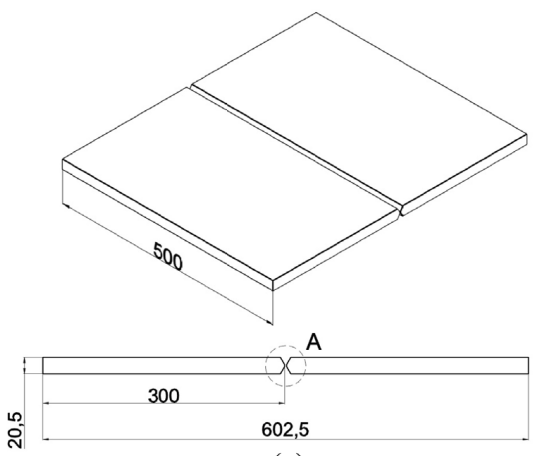

(a)

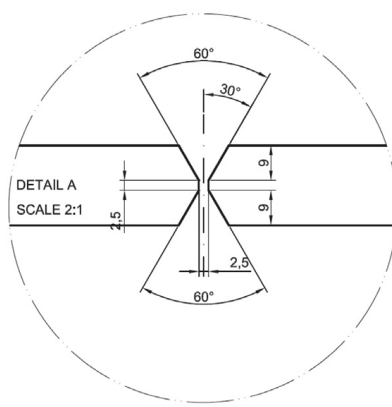

(b)

Figure 1: (a) Dimensions of plates for welding and (b) detail of the bevel adopted for the joint.

Table 2: Chemical composition and mechanical properties of the consumable for the root pass.

\begin{tabular}{|c|c|c|c|c|c|}
\hline Consumable & \multicolumn{5}{|c|}{ Chemical composition (wt\%) } \\
\hline \multirow{7}{*}{ E71T-1 } & $\mathrm{C}$ & $\mathrm{Mn}$ & $\mathrm{Si}$ & $\mathrm{S}$ & $\mathrm{P}$ \\
\hline & 0,12 & 1,75 & 0,9 & 0,03 & 0,03 \\
\hline & \multicolumn{5}{|c|}{ Mechanical properties } \\
\hline & \multicolumn{3}{|c|}{ Yield stress (MPa) } & \multicolumn{2}{|r|}{390} \\
\hline & \multicolumn{3}{|c|}{ Ultimate strength (MPa) } & \multicolumn{2}{|r|}{$490-670$} \\
\hline & \multicolumn{3}{|c|}{ Elongation (\%) } & \multicolumn{2}{|r|}{22} \\
\hline & \multicolumn{3}{|c|}{ Absorbed energy $(\mathrm{J})$ in $-27^{\circ} \mathrm{C}$} & \multicolumn{2}{|r|}{27} \\
\hline
\end{tabular}

Table 3: Chemical composition and mechanical properties of the flux and electrode for SAW.

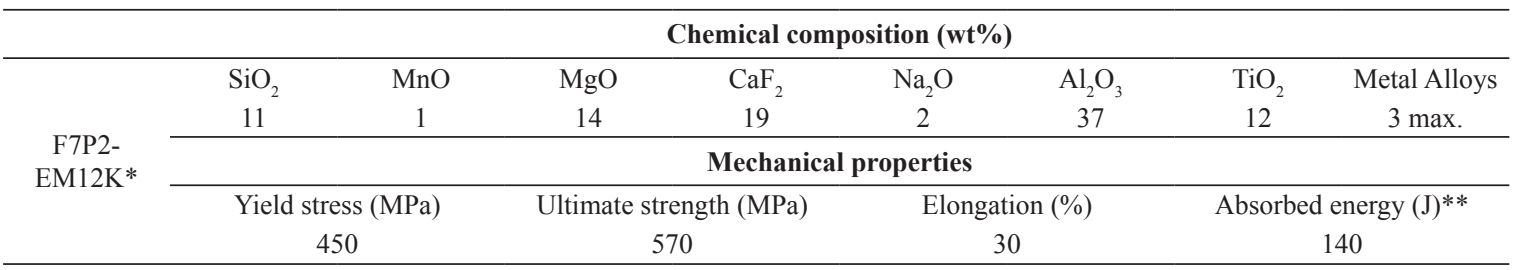

*In accordance with AWS A5/17.23, **Carried out at $-29^{\circ} \mathrm{C}$

Table 4: Welding parameters for one wire SAW (S-SAW).

\begin{tabular}{lccccc}
\hline \multicolumn{1}{c}{ Pass number } & $\mathbf{1}$ & $\mathbf{2}$ & $\mathbf{3}$ & $\mathbf{4}$ & $\mathbf{5}$ \\
\hline \multicolumn{1}{c}{ Welding Process } & FCAW & SAW (DC) & SAW (DC) & SAW (DC) & SAW (DC) \\
\hline Arc voltage (V) & 27.0 & 34.0 & 34.5 & 34.0 & 32.0 \\
Current (A) & 140 & 468 & 420 & 620 & 650 \\
Travel speed (mm/min) & 214.2 & 329.0 & 303.6 & 325.8 & 237.0 \\
Efficiency & 0.80 & 0.95 & 0.95 & 0.95 & 0.95 \\
Heat input (kJ/mm) & 0.84 & 2.75 & 2.75 & 3.71 & 5.00 \\
\hline
\end{tabular}


Microhardness testing was performed in accordance with ASTM E384 ${ }^{9}$, and conducted using a LECO's LM Series microhardness testing machine with a $300 \mathrm{gf}$ load and a hold time of $10 \mathrm{~s}$. A $0.2 \mathrm{~mm}$ grid spacing was used, revealing the distribution and individual hardness values in selected regions of the weld joints, as depicted in Figure 2. Charpy V impact fracture toughness tests were performed according to ASTM E23 standard ${ }^{10}$ for the base metal and the weld metal. Tests were done for three samples each at $-60,-40,-20,0$ and $25^{\circ} \mathrm{C}$. Samples from the base metal were taken from the longitudinal rolling direction (LRD) and transverse rolling direction (TRD).

\section{Results and Discussion}

\section{Microstructural characterization}

Microstructural characterization of the joints is shown in Figure 3 to 8 . Figure 3 .a presents the microstructure of the base metal. It can be seen to be ferrite and (F) and pearlite (P). Table 6 shows a comparison between volumetric fractions of the $\mathrm{F}$ and $\mathrm{P}$ in the HAZ and BM.

Figure 3.b shows the microstructure of the base metal etched with Klemm I reagent. This reagent is able to show ferrite as blue or brown, depending upon the crystallographic orientation. In addition, it identifies the presence of MA

Table 5: Welding parameters for two wires tandem SAW (T-SAW)

\begin{tabular}{lccccc}
\hline \multicolumn{1}{c}{ Pass number } & $\mathbf{1}$ & \multicolumn{2}{c}{$\mathbf{3}$} \\
\hline \multicolumn{1}{c}{ Welding Process } & FCAW & SAW-DC & SAW-AC & SAW-DC & SAW-AC \\
\hline Arc voltage (V) & 27.0 & 29.5 & 32.0 & 29.0 & 33.0 \\
Current (A) & 140 & 620 & 540 & 620 & 540 \\
Travel speed (mm/min) & 205.2 & 530.0 & 530.0 & 530.0 & 484.8 \\
Efficiency & 0.80 & 0.95 & 0.095 & 0.95 & 0.95 \\
Heat input (kJ/mm) & 0.88 & $1.98^{*}$ & $1.85^{*}$ & $1.94^{*}$ & $2.09^{*}$ \\
\hline
\end{tabular}

$(*)$ The heat input of each wire was added per pass.

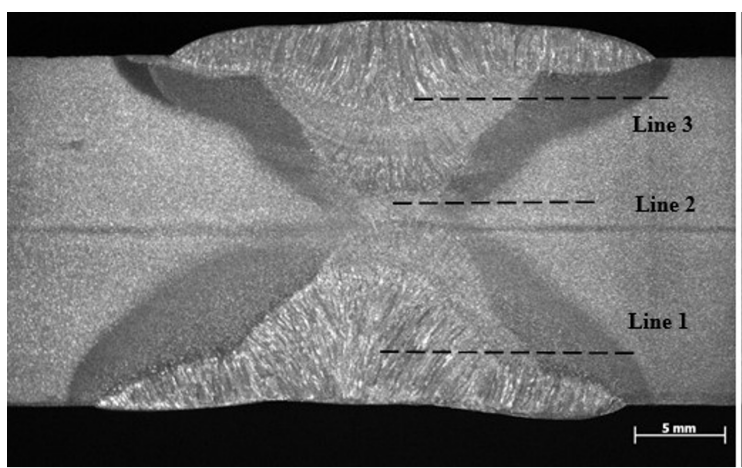

(a)

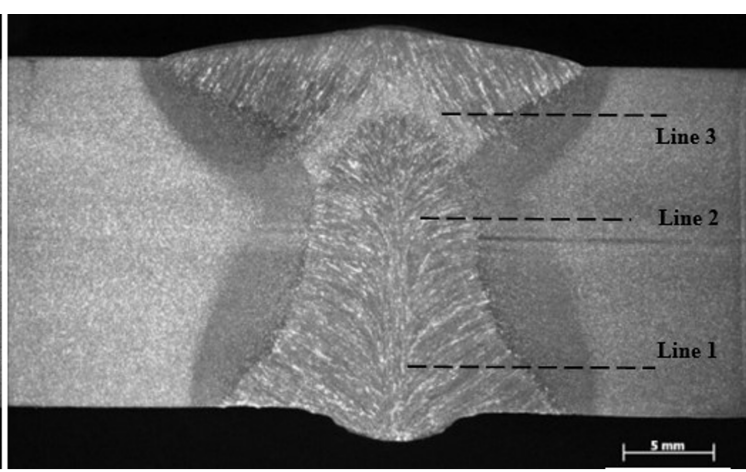

(b)

Figure 2: Weld joints showing the regions of microhardness indentations for (a) EXP1 (single wire S-SAW) and (b) EXP 2 (two wires T-SAW).
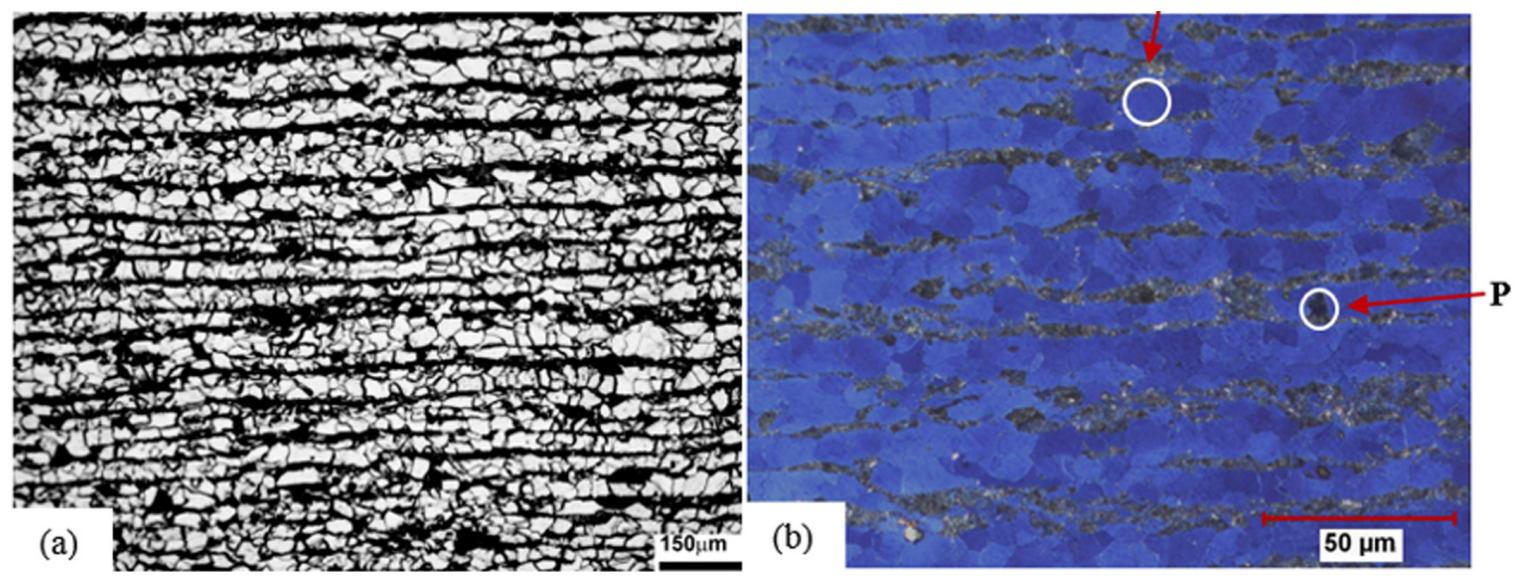

Figure 3: Optical microstructure characterization of base metal. (a) 2\% Nital etching and (b) Klemm I etching. 


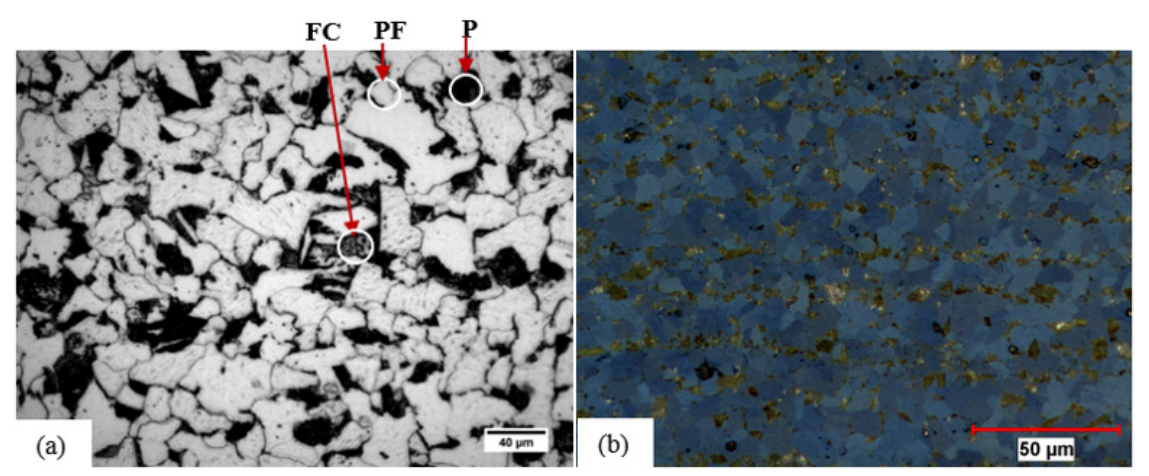

Figure 4: Optical microstructure characterization of FGHAZ for S-SAW. (a) 2\% Nital etching and (b) Klemm I etching.

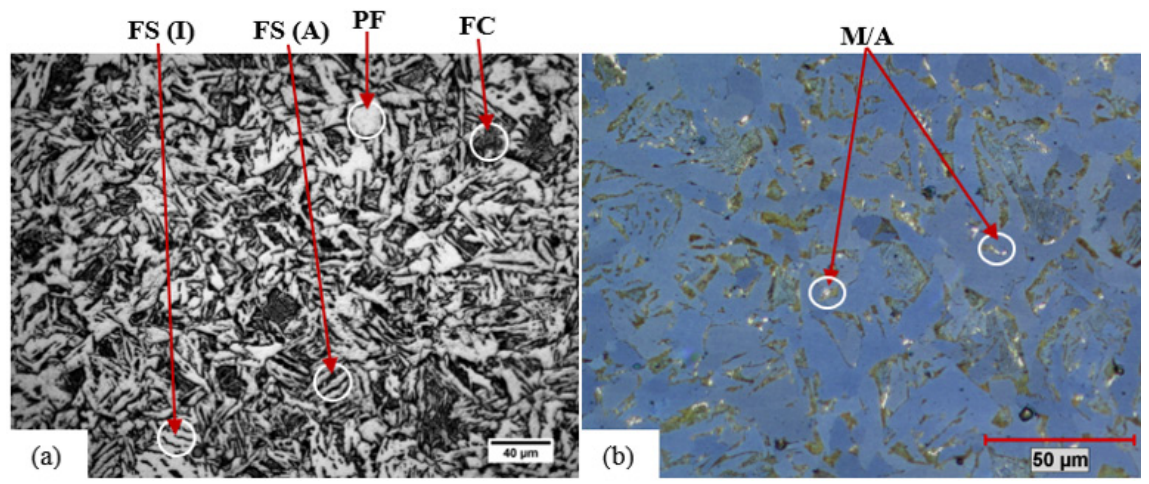

Figure 5: Optical microstructure characterization of CGHAZ for S-SAW. (a) 2\% Nital etching and (b) Klemm I etching.

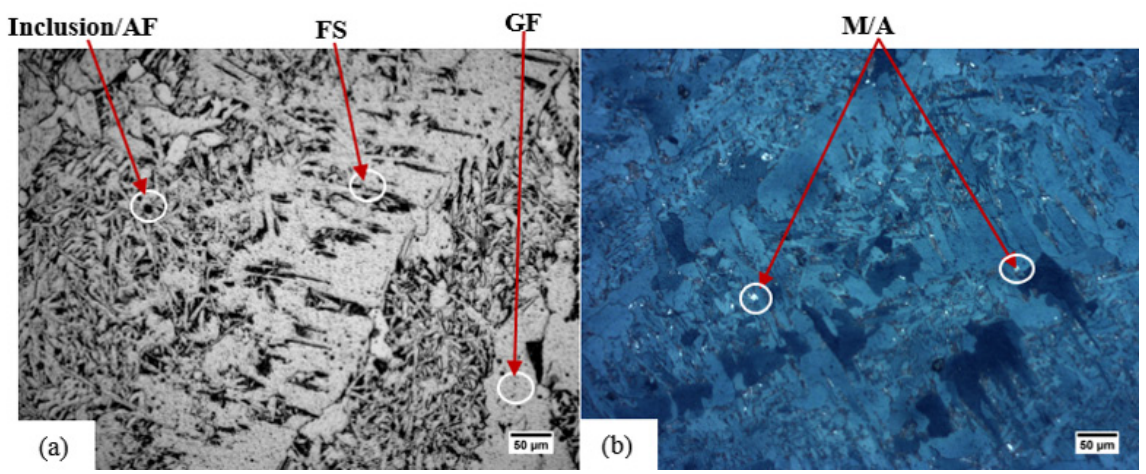

Figure 6: Optical microstructure characterization of weld metal (WM) for S-SAW. (a) 2\% Nital etching and (b) Klemm I etching.

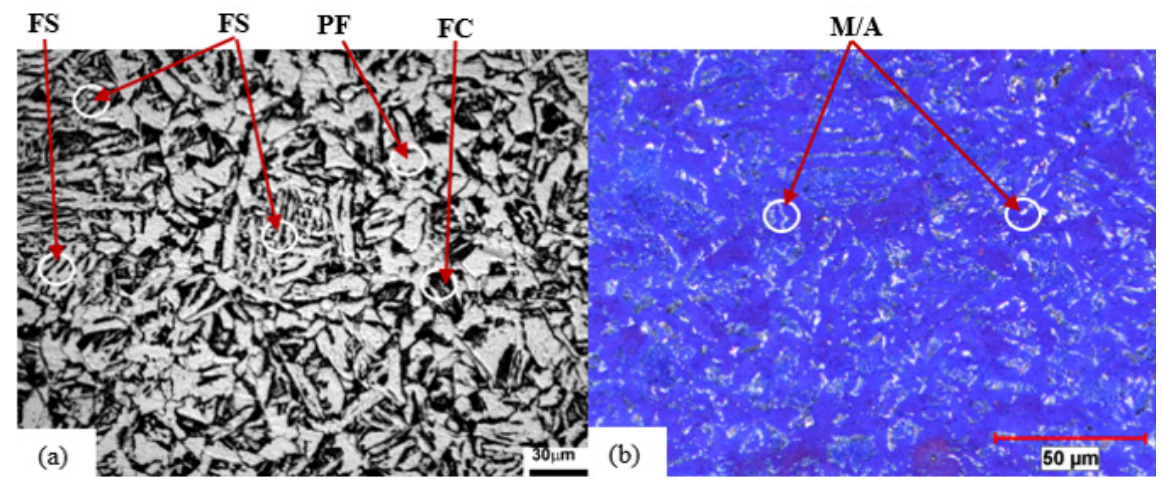

Figure 7: Optical microstructure characterization of CGHAZ for T-SAW. (a) 2\% Nital etching and (b) Klemm I etching. 


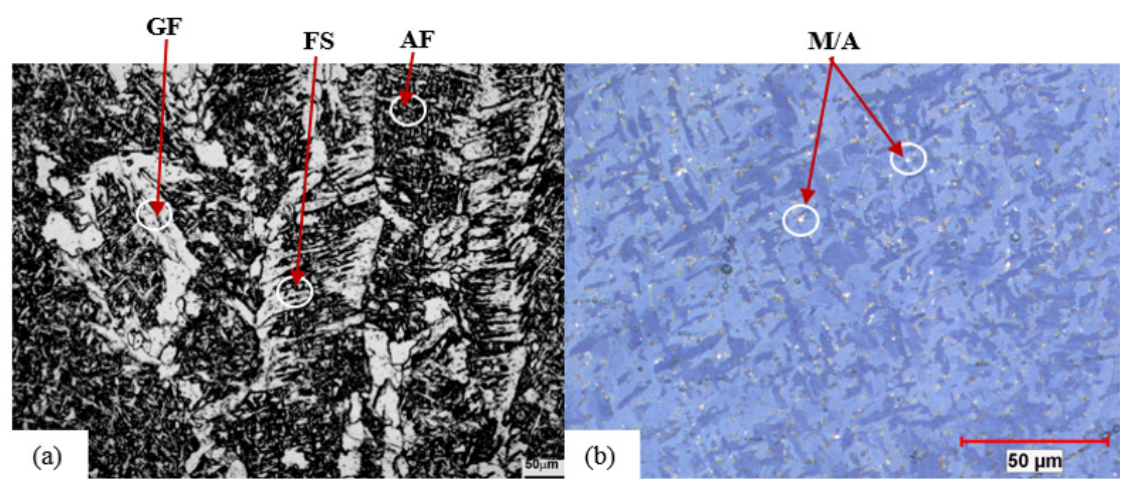

Figure 8: Optical microstructure characterization of weld metal (WM) for T-SAW. (a) 2\% Nital etching and (b) Klemm I etching.

Table 6: Volume fractions of the HAZ regions compared with the volumetric fraction of the base metal (BM).

\begin{tabular}{lccc}
\hline \multicolumn{1}{c}{ Experiment } & HAZ region & $\begin{array}{c}\text { Volume Fraction of } \\
\text { ferrite (\%) }\end{array}$ & $\begin{array}{c}\text { Change of the volumetric } \\
\text { fraction related to BM (\%) }\end{array}$ \\
\hline S-SAW (one wire) & FG & 62.2 & 15.9 \\
T-SAW (two wires) & (Fine grain) & 71.2 & 3.8 \\
S-SAW (one wire) & CG & 57.5 & 22.3 \\
T-SAW (two wires) & (Coarse grain ) & 66.5 & 10.1 \\
\hline
\end{tabular}

microconstituents as white regions. In this image, there is only ferrite in blue and pearlite as dark gray. The grain size in the base metal was measured. The results show a range from 8 to $17 \mu \mathrm{m}$, which agrees with past work ${ }^{11}$. Specifically, the ferrite grain size and the banded structure are due to microstructural changes that occur during TMCP processing.

Figure 4.a presents the microstructure in the FGHAZ region (fine grain heat affected zone) in the welded joint for S-SAW. The observed constituents are polygonal ferrite (PF), ferrite with non-lamellar carbides (FC) and perlite (P). The mean grain size for this region was between 10 and $12 \mu \mathrm{m}$, which is slightly lower than that for the base metal. The temperature during the welding process is high enough to allow partial austenite transformation in the intercritical region. However, the region from the FGHAZ to the fusion line does not undergo significant grain growth of austenite, since the cooling rate drives the austenitic transformation in polygonal ferrite grains ${ }^{12}$. Likewise, the FGHAZ was etched with Klemm I reagent (Figure 4.b) and no MA microconstituents are present. The large amount of $\mathrm{PF}$ in the FGHAZ occurs because the cooling rate in this region is lower than the cooling rate for the CGHAZ region (coarse grain heat affected zone). A cooling rate between 1 and $5^{\circ} \mathrm{C} / \mathrm{s}$ in the $\mathrm{AH} 36$ steel promotes the formation of polygonal ferrite and perlite ${ }^{13}$.

The microstructure in the CGHAZ for S-SAW (Figure 5.a) was composed of grain boundary ferrite (GF), ferrite with a second phase FS (aligned FS(A) and nonaligned FS(NA)), polygonal ferrite $(\mathrm{PF})$ and ferrite with non-lamellar carbides (FC). These microstructural changes occur when the temperature in the CGHAZ region is above the $\mathrm{A}_{\mathrm{c} 3}$ temperature for AH36 steel, thus promoting the growth of austenite grains. If the peak temperature during the last pass exceeds the $\mathrm{A}_{c 3}$ temperature, the resulting austenite after cooling may decompose in Widmanstätten ferrite, bainite and MA microconstituents, which can be seen in Figure 5.b. as white dots.

Figure 6 depicts the weld metal microstructure for S-SAW. Figure 6.a shows acicular ferrite (AF), ferrite with second phase FS (aligned FS(A) and nonaligned FS(NA)) and grain boundary ferrite (GF). In Figure 6.b one can see the MA microconstituents with a volumetric fraction comparable to the CGHAZ of S-SAW.

The microstructure presented in Figure 7 is similar to Figure 5. The difference is a coarser microstructure and the amount of MA microconstituent, which is larger for T-SAW. These results are likely due to the higher heat input used for T-SAW, which produced higher peak temperatures for comparable thermal cycles. These characteristics in the thermal cycles produced the changes in the microstructure observed.

Figure 8 shows the weld metal microstructure for T-SAW. The phases are identical to those in Figure 6. In Figure 8.b one can see the MA microconstituents with a volumetric fraction higher than that for the WM (weld metal) of S-SAW.

The MA microconstituent is associated with the dissolution of carbides and a decrease in the $\mathrm{M}_{\mathrm{s}}$ and $\mathrm{M}_{\mathrm{f}}$ temperatures. The amount of retained austenite depends on the cooling rate of the joint; the faster the cooling rate, the larger the volumetric fraction of austenite. This was observed for the case of T-SAW.

The volumetric fraction of MA microconstituent was measured by the colored etching technique. The results are presented in Figure 9. However, this metallographic technique is not sufficient to prove, in a precise way, the presence of austenite. Thus, X-ray diffraction was used to identify austenite, martensite and ferrite phases in the HAZ of the welded joint made by S-SAW and T-SAW. This technique identifies phases with at least $5 \%$ in volume fraction. X-ray diffraction was carried out in the CGHAZ regions, which according to image analysis had higher volume fractions of 
MA. The regions showed the presence of ferrite, austenite and martensite. Table 7 and 8 present the identification of the planes of each phase with higher intensity. The results of the diffraction patterns are depicted in Figure 10 and Figure 11 .

The calculations of the crystal parameters of the phases found in the diffraction patterns are presented in Table 9, to verify the planes and phases identified in Table 7 and Table 8 . The analysis by X-ray diffraction confirmed the presence of retained austenite and martensite phases in the experiments under study; in other words, the presence of MA microconstituent.

The volume fractions of the MA microconstituents for S-SAW and T-SAW, for the separate weld and HAZ regions, are shown in Figure 9. There is a larger quantity of MA in

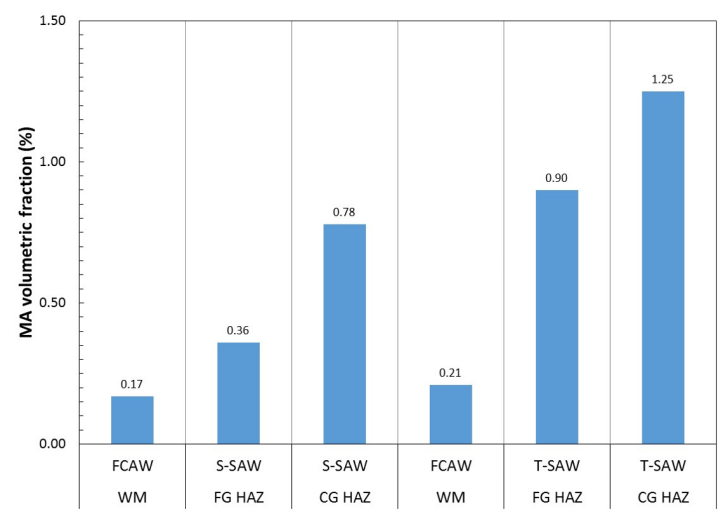

Figure 9: Volume fraction of MA microconstituent present in the WM and regions of the HAZ for the two experiments, S-SAW and T-SAW. the CGHAZ for the two welding conditions, with the T-SAW sample having a fraction about $60 \%$ higher than the S-SAW sample. It is possible that the higher heat input in T-SAW is responsible for this result. Figure 12 shows the heat input for each pass. The higher the heat input, the higher the peak temperature at a location in the HAZ. In addition, the cooling rate is slower, allowing more time at a given temperature in the HAZ. This increases the grain size of the regions which, together with carbide dissolution, increases the hardenability of the region in the HAZ. As a consequence, the $\mathrm{M}_{\mathrm{s}}$ and $\mathrm{M}_{\mathrm{f}}$ temperatures decrease, and more retained austenite is formed together with untempered martensite.

\section{Microhardness test}

Figures 13 to 15 present a microhardness profiles for lines 1, 2 and 3, respectively, in Figure 2. Line 1, in Figure 13, corresponds to the filling pass and line 3 , in Figure 15, is the finishing pass region. Figure 13 shows that the values of microhardness along line 1 in S-SAW are similar to line 1 in T-SAW. In both cases, reheating due to subsequent passes can produce a kind of a "localized heat treatment", keeping both hardness profiles almost the same.

By comparing S-SAW and T-SAW, it is possible to observe significant differences in microhardness values, in particular in the CGHAZ. Besides the heat input, the values of microhardness vary due to the different microstructures present in the welded joint and due to reheating thermal cycles during welding, which act as a kind of non-isothermal heat treatment.

Figure 14 shows the results of the Vickers microhardness measurements for line 2, the root pass for S-SAW and T-SAW. The difference in microhardness values are due to the peak temperature, which is a function of the heat input, (Figure 12).

Table 7: Phases identified by X-rays diffraction in the CGHAZ region of one wire SAW joint (S-SAW).

\begin{tabular}{cccc}
\hline Angle (degree) & $\begin{array}{c}\text { Distance of crystal } \\
\text { plane (Angstroms) }\end{array}$ & Phase & Plane \\
\hline 42,8291 & 2,115 & austenite & 111 \\
44,7742 & 2,02251 & martensite & 101 \\
44,8983 & 2,02222 & ferrite & 110 \\
45,197 & 2,0045 & martensite & 200 \\
50,776 & 1,7966 & austenite & 200 \\
65,2866 & 1,4316 & ferrite & 211 \\
\hline
\end{tabular}

(*) Identification in the diffraction pattern background

Table 8: Phases identified by X-ray diffraction in the CGHAZ region of two wires SAW joint (T-SAW).

\begin{tabular}{|c|c|c|c|c|}
\hline Angle (degree) & $\begin{array}{l}\text { Distance of crystal } \\
\text { plane (Angstroms) }\end{array}$ & Phase & Plane & Note \\
\hline 42,757 & 2,1131 & austenite & 111 & * \\
\hline 44,058 & 2,0537 & martensite & 101 & \\
\hline 44,944 & 2,0153 & ferrite & 110 & \\
\hline 45,514 & 1,9913 & martensite & 110 & $*$ \\
\hline 50,653 & 1,8007 & austenite & 200 & $*$ \\
\hline 65,338 & 1,4270 & ferrite & 200 & \\
\hline 82,419 & 1,1692 & ferrite & 211 & \\
\hline
\end{tabular}




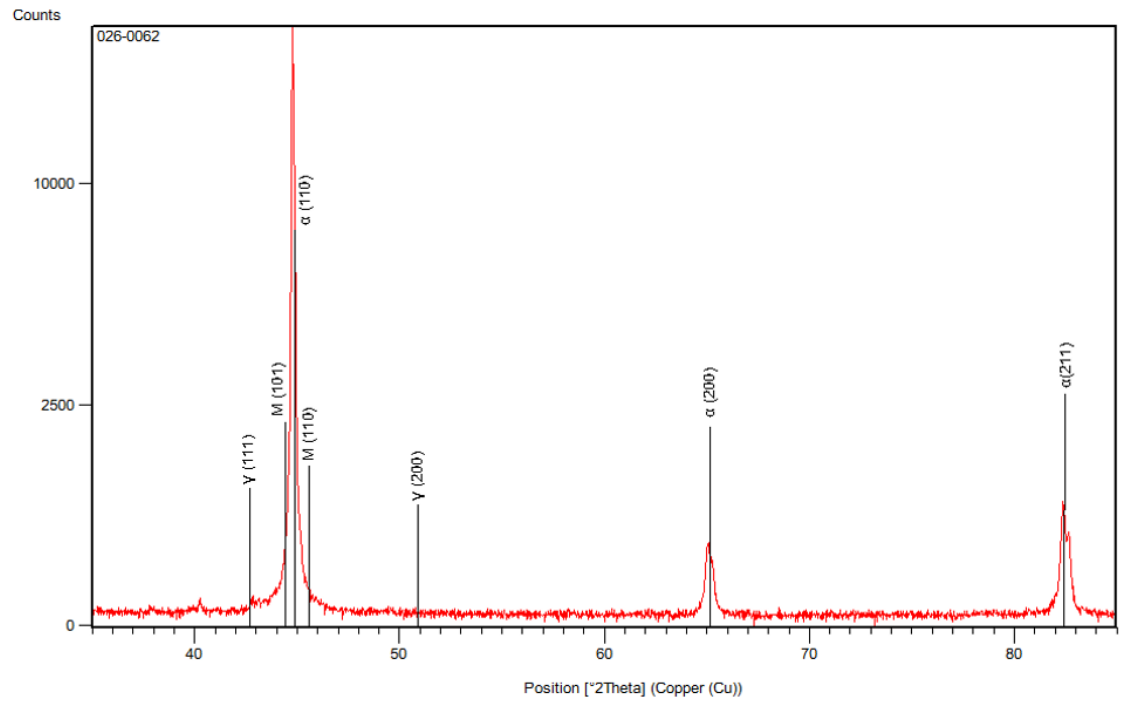

Figure 10: Diffraction pattern for sample welded by S-SAW.

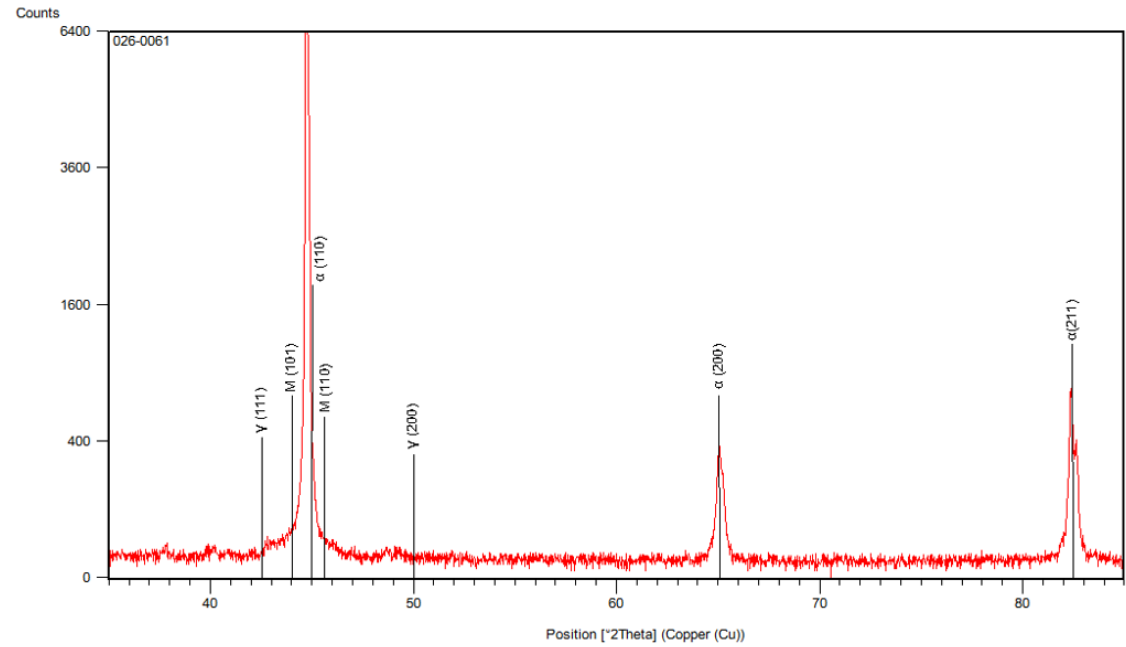

Figure 11: Diffraction pattern for sample welded by T-SAW.

Table 9: Crystal parameters for the identified phases by X-ray diffraction.

\begin{tabular}{|c|c|c|c|c|c|c|}
\hline \multirow[b]{2}{*}{ Phase } & \multirow{2}{*}{$\begin{array}{c}\text { Crystal } \\
\text { structure }\end{array}$} & & \multicolumn{4}{|c|}{ Crystal Parameter (Angstrons) } \\
\hline & & & $\begin{array}{l}\text { JPDS Chart } \\
\text { Identification }\end{array}$ & JPDS & S-SAW & T-SAW \\
\hline Ferrite & bcc & $\mathrm{a}$ & $06-0696$ & 2.8664 & 2.85 & 2.86 \\
\hline \multirow[t]{2}{*}{ Austenite } & fcc & $\mathrm{a}$ & $52-0513$ & 3.6599 & 3.68 & 3.69 \\
\hline & & $\mathrm{a}$ & & 2.859 & 2.82 & 2.83 \\
\hline \multirow[t]{2}{*}{ Martensite } & tetragonal & $\mathrm{c}$ & $44-1290$ & 2.937 & 3.00 & 2.89 \\
\hline & & $\mathrm{c} / \mathrm{a}$ & & 1.0273 & 1.06 & 1.02 \\
\hline
\end{tabular}

\section{Charpy V notch impact test}

The results obtained in the Charpy V notch impact test are depicted in Figure 16. An ANOVA analysis, with 95\% confidence, showed no difference in weld metal absorbed energy, comparing samples from the two conditions (S-SAW and T-SAW). However, when comparing different regions of the welded joint, the weld metal had the lowest impact toughness. This deleterious behavior is due to an as-solidified weld metal with a coarse grain size and also the presence of microsegregation. ${ }^{14}$

According to the fracture images obtained through scanning electron microscopy, shown in Figure 17, it is possible to identify transgranular fractures and dimples. This is the typical appearance of specimens that suffered ductile 


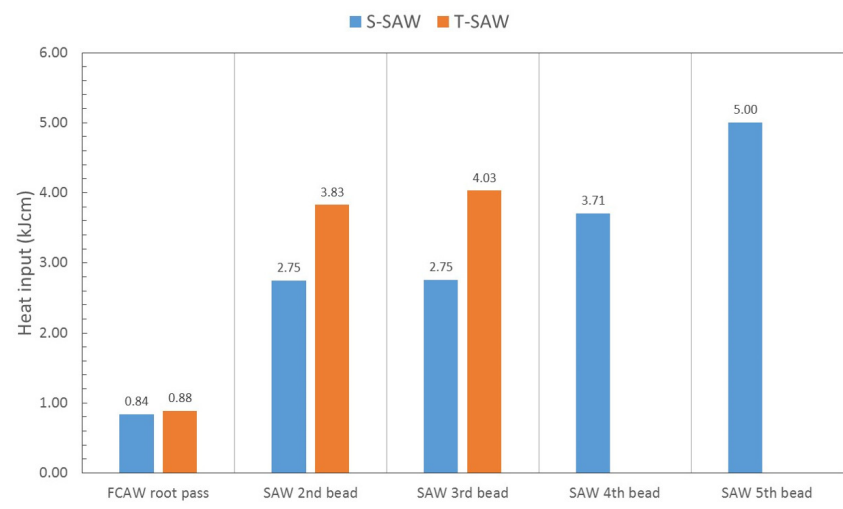

Figure 12: Heat input for each pass in S-SAW and T-SAW experiments.

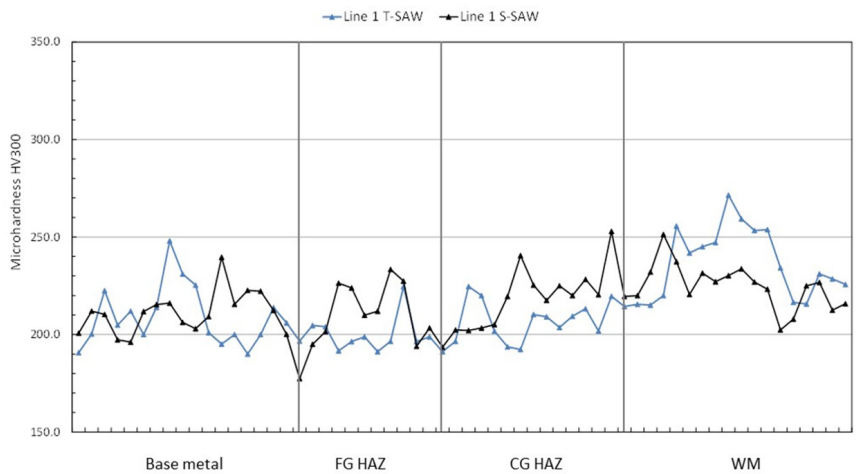

Figure 13: Results of microhardness for line 1, as presented in Figure 2.

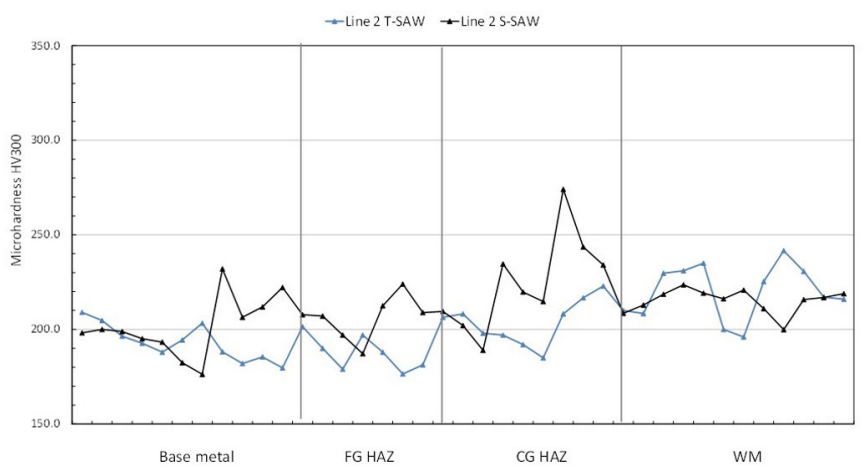

Figure 14: Results of microhardness for line 2, as presented in Figure 2.

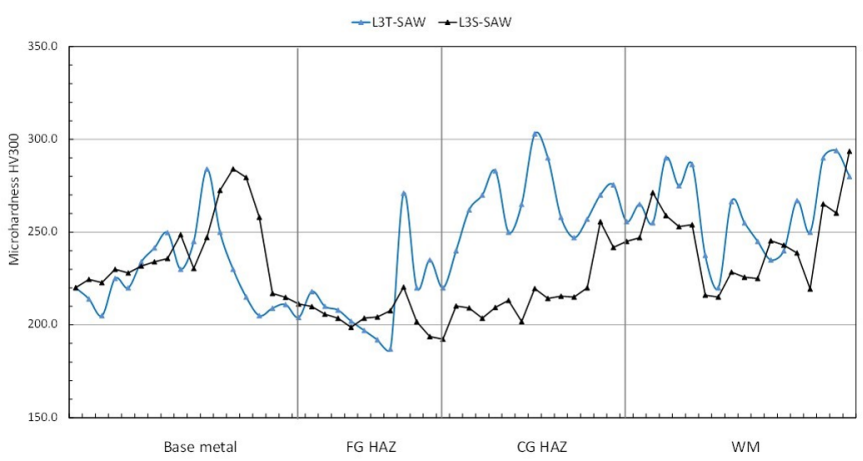

Figure 15: Results of microhardness for line 3, as presented in Figure 2. 


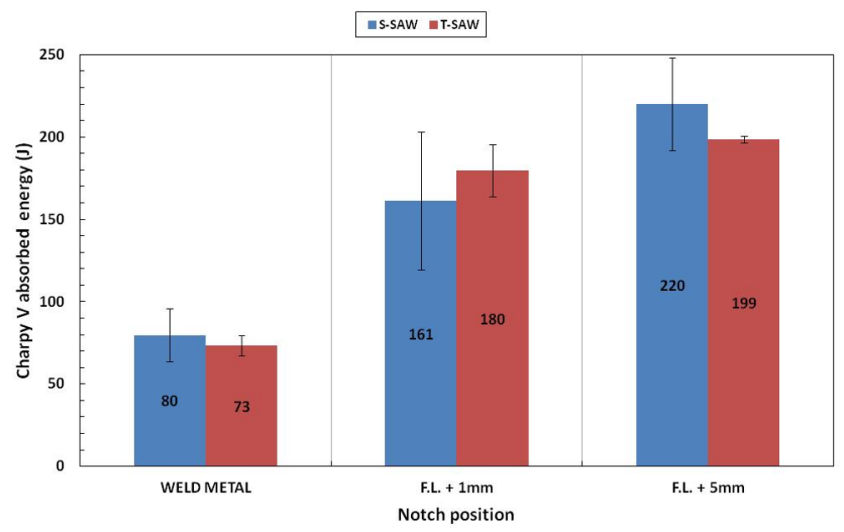

Figure 16: Results of Charpy $\mathrm{V}$ tests at $0^{\circ} \mathrm{C}$ for different regions of the welded joint; weld metal, $1 \mathrm{~mm}$ away from the fusion line and $5 \mathrm{~mm}$ from the fusion line.
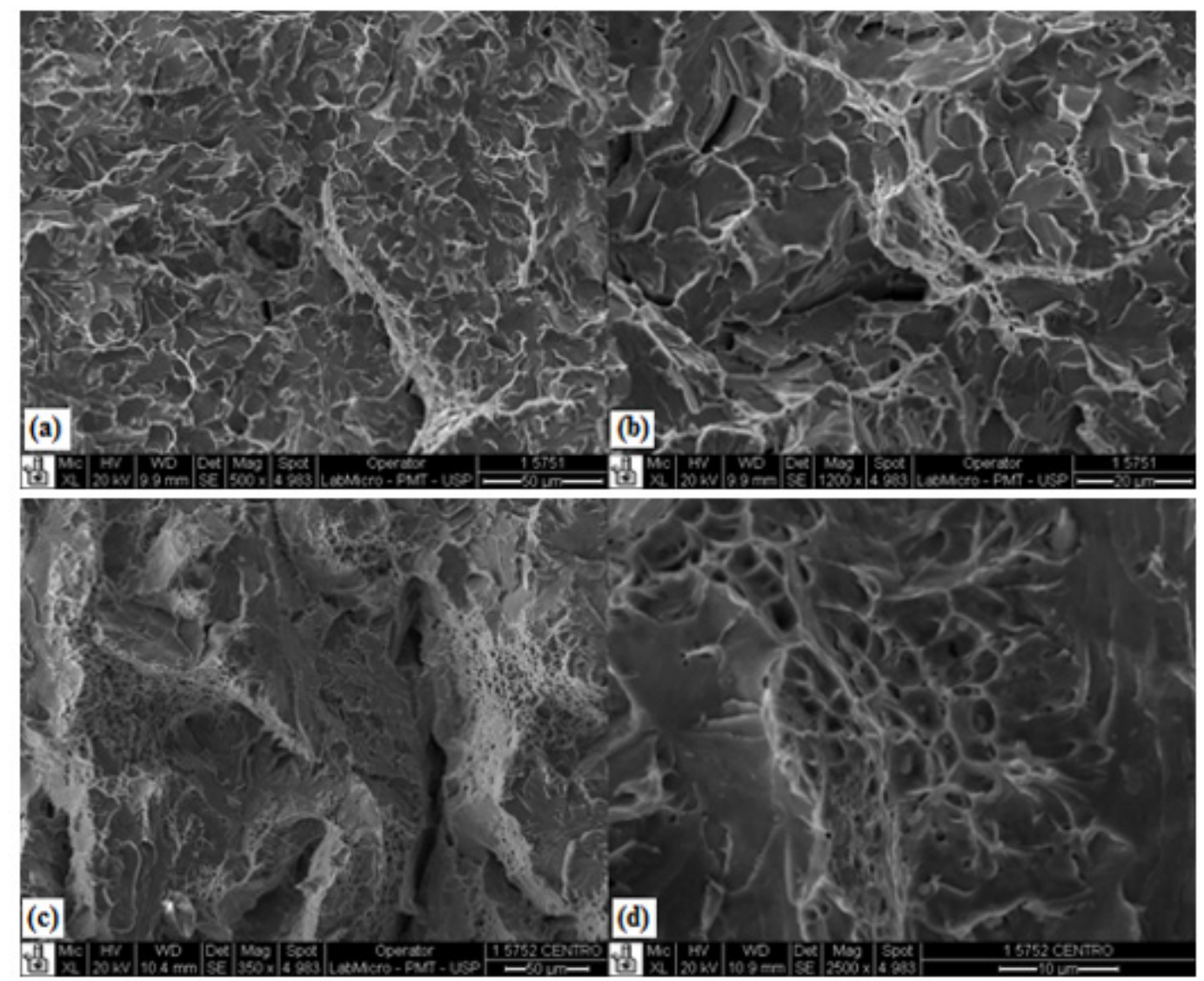

Figure 17: (a) and (b) SEM fractographs of Charpy samples at $0^{\circ} \mathrm{C}$ of the weld metal for S-SAW; (c) and (d) SEM fractographs of Charpy fractographs at $0^{\circ} \mathrm{C}$ of the weld metal for T-SAW.

fracture. The Charpy impact test, for a notch situated $5 \mathrm{~mm}$ away from fusion line $(\mathrm{FL}+5)$, offers the highest average value of absorbed energy $(220 \mathrm{~J})$ in the FG HAZ region for $\mathrm{S}$-SAW. That is a zone of grain refinement, which underwent reheating by subsequent welding passes. The average grain size is $5.5 \mu \mathrm{m}$ and the predominant microstructure consists of polygonal ferrite with a smaller fraction of pearlite. The same zone observed in T-SAW not only consists of polygonal ferrite and pearlite, but also a microstructure with zones of Widmanstätten ferrite (WF). The presence of those morphologies has contributed to a slight reduction in the amount of absorbed energy, which is about $10 \%$ lower than that for S-SAW.

The results of the ANOVA analysis, with $95 \%$ confidence, presented no difference between the absorbed energy values in the Charpy impact test for the region situated $1 \mathrm{~mm}$ 
away from the fusion line towards the HAZ $(\mathrm{FL}+1)$ for both S-SAW and T-SAW. The same results were observed when comparing zones $(\mathrm{FL}+5)$ and $(\mathrm{FL}+1)$, i.e., both experiments presented no difference in absorbed energy values. These results can be confirmed when comparing the $(\mathrm{FL}+1)$ zone microstructure, which corresponds to the CGHAZ, to the $(\mathrm{FL}+5)$ zone microstructure, which corresponds to the FGHAZ. The microstructural changes for both experiments are virtually the same. It can be noticed by means of microstructural characterization that the $(\mathrm{FL}+1)$ zone for both experiments have MA constituents, which does not occur in the $(\mathrm{LF}+5) \mathrm{FGHAZ}$ zone.

\section{Conclusions}

Based on the aforementioned experiments and their respective results, we may draw the following conclusions:

1. The microstructure of the HAZ for both experiments includes polygonal ferrite, grain boundary ferrite, ferrite with second aligned/non-aligned phase, ferrite with carbides and martensite/austenite microconstituents. In particular, the CGHAZ region in the T-SAW (tandem welding technique) has more MA greater than the same region in S-SAW (single wire condition).

2. The microhardness values for the regions analyzed fluctuate, not only because of the phases and inclusions present, but also because of the different heat input used in each pass. This is the case of the filling pass for T-SAW (line 3 in Figure 2) condition with a hardness of $240 \mathrm{HV}$. However, the heat input

\section{References}

1. Tsukada K, Watanabe, I. The progress of thermo-mechanical control process for HSLA plate in Japan. Key Engineering Materials. 1993;84-85:22-26.

2. Shiga C, Amano K, Enami T. Application of Multipurpose accelerated cooling systems (MACS) to the production of HSLA steel plate. In: International Conference on Technology and application of High Strength Low Alloy (HSLA) Steels Proceedings. Philadelphia: American Society for Metals; 1984.643 -649 .

3. Pickering FB. Physical metallurgy and the design of steels. London: Applied Science; 1983.

4. Bohme D, Nentwig AW, Knoch R. A high efficiency welding process in double wire welding. In: II Welding International Congress. Proceedings. Auckland; 1996. p 1393 - 1407.

5. Almqvist I, Gronbeck SB. Submerged arc welding. 2nd ed. Cambridge: The Welding Institute; 1978.

6. American Society for Testing and Materials. ASTM A131/ A131M-08: Standard Specification for Structural Steel for Ships. ASTM International; 2001.

7. AWS CODE A5. 20/A5.20M. Carbon steel electrodes for flux cored arc welding [Internet]. American National Standard; for the pass corresponding to line 3 in the T-SAW was 33\% lower than that in single wire condition (S-SAW). The CGHAZ region in T-SAW was harder than the one in S-SAW.

3. The weld metal for both conditions reached an average absorbed energy value $63 \%$ lower then the energy of the grain refined and the base metal regions. In summary, two factors are associated with this deleterious behavior in the weld metal (WM). The first one is the microstructural characteristics of the weld metal, which consists AF, GF and FS, where the crack follows the grain boundaries of FG and spreads easily between FS plates separated by low angle. The second one is the fracture pattern. Fractography of the weld metal exhibit transgranular fractures and dimples.

4. According to the ANOVA analysis, the CGHAZ for T-SAW had the same absorbed energy as S-SAW, in the same notch position in the HAZ regions.

\section{Acknowledgements}

The authors wish to acknowledge funding provided by the Emerging Leaders in the Americas Program (ELAP) and the Natural Science and Engineering Research Council of Canada. The use of research facilities at EVRAZ Inc. NA as well as the supply of steel for this research from Usiminas Company at Cubatão - SP are also acknowledged. Finally, the assistance of EVRAZ Inc. NA staff, especially Dr. Laurie Collins, Muhammad Rashid, and Jon Jackson in carrying out some of the test work is also acknowledged.

2005. [cited 2015 Jul 21]. Available from: https://pubs.aws. org/Download_PDFS/A5.20-A5.20M-2005PV.pdf

8. Grong Ø. Metallurgical modelling of welding. 2nd ed. London: Institute of Materials; 1997.

9. ASTM E384. Standard Test Method for Knoop and Vickers Hardness of Materials [Internet]. ASTM International;2005. [cited 2015 Jul 21]. Available fron: http://www.astm.org/ FULL_TEXT/E384/HTML/E384.htm.

10. ASTM International. ASTM E23; 2006.

11. Rui F, Zhang R, Sheng LI, Kong G, Zhu X. Study on forming mechanism of lamination defect of AH36 shipbuilding plate steel. Advanced Materials Research. 2012;562-564:106-110.

12. Poorhaydari K, Ivey DG. Estimation of cooling in the welding plates with thickness Intermediate. Welding Journal. 2005;84:149155.

13. Ghosh SD, Chatterjee S, Ramachandrarao P. Microstructural characterization of controlled forged HSLA -80 steel by transmission electron microscopy. Materials Characterization. 2003;50:305-315.

14. Svensson LE. Control of microstructures and properties in steel arc welds. Cambridge - U.K: Materials Science \& Technology; 1993. 233 p. 